\title{
Blood Urea Nitrogen to Serum Albumin Ratio (BAR) Predicts Critical Illness in Patients with Coronavirus Disease 2019 (COVID-19)
}

\author{
Dong Huang' \\ Huan Yang' \\ $\mathrm{He} \mathrm{Yu}^{\prime}$ \\ Ting Wang' \\ Zhu Chen ${ }^{2}$ \\ Zongan Liang $\left.{ }^{\prime}\right)^{\prime}$ \\ Rong $\mathrm{YaO}^{3}$ \\ 'Department of Respiratory and Critical \\ Care Medicine, West China Hospital, \\ Sichuan University, Chengdu, Sichuan, \\ People's Republic of China; ${ }^{2}$ Department \\ of Infectional Inpatient Ward Two, \\ Chengdu Public Health Clinical Medical \\ Center, Chengdu, Sichuan, People's \\ Republic of China; ${ }^{3}$ Department of \\ Emergency Medicine, Emergency Medical \\ Laboratory, West China Hospital, Sichuan \\ University, Chengdu, Sichuan, People's \\ Republic of China
}

Correspondence: Zongan Liang Department of Respiratory and Critical Care Medicine, West China Hospital, Sichuan University, Chengdu, Sichuan,

People's Republic of China

Tel +86028 85422377

Email liangza@scu.edu.cn

Rong Yao

Department of Emergency Medicine, Emergency Medical Laboratory, West China Hospital, Sichuan University, Chengdu, Sichuan, People's Republic of China

Tel +860288542 246I

Email yaorong@wchscu.cn
Purpose: We sought to explore the prognostic value of blood urea nitrogen (BUN) to serum albumin ratio (BAR) and further develop a prediction model for critical illness in COVID-19 patients.

Patients and Methods: This was a retrospective, multicenter, observational study on adult hospitalized COVID-19 patients from three provinces in China between January 14 and March 9, 2020. Primary outcome was critical illness, including admission to the intensive care unit (ICU), need for invasive mechanical ventilation (IMV), or death. Clinical data were collected within 24 hours after admission to hospitals. The predictive performance of BAR was tested by multivariate logistic regression analysis and receiver operating characteristic (ROC) curve and then a nomogram was developed.

Results: A total of 1370 patients with COVID-19 were included and 113 (8.2\%) patients eventually developed critical illness in the study. Baseline age (OR: 1.031, 95\% CI: 1.014, 1.049), respiratory rate (OR: 1.063, 95\% CI: 1.009, 1.120), unconsciousness (OR: 40.078, 95\% CI: 5.992, 268.061), lymphocyte counts (OR: 0.352, 95\% CI: 0.204, 0.607), total bilirubin (OR: 1.030, 95\% CI: 1.001, 1.060) and BAR (OR: 1.319, 95\% CI: 1.183, 1.471) were independent risk factors for critical illness. The predictive AUC of BAR was 0.821 (95\% CI: $0.784,0.858 ; \mathrm{P}<0.01$ ) and the optimal cut-off value of BAR was $3.7887 \mathrm{mg} / \mathrm{g}$ (sensitivity: 0.690 , specificity: 0.786 ; positive predictive value: 0.225 , negative predictive value: 0.966 ; positive likelihood ratio: 3.226 , negative likelihood ratio: 0.394 ). The $\mathrm{C}$ index of nomogram including above six predictors was 0.9031125 (95\% CI: 0.8720542 , 0.9341708).

Conclusion: Elevated BAR at admission is an independent risk factor for critical illness of COVID-19. The novel predictive nomogram including BAR has superior predictive performance.

Keywords: blood urea nitrogen, albumin, predictive performance, critical illness, COVID-19

\section{Introduction}

The highly contagious coronavirus disease 2019 (COVID-19), caused by severe acute respiratory syndrome coronavirus 2 (SARS-CoV-2) infection, has swept the world at an unprecedented rate since December 2019. ${ }^{1}$ The World Health Organization (WHO) officially classified the COVID-19 outbreak to be a pandemic in March 2020. ${ }^{2}$ At the time of this writing, there were more than 120 million known cases and 2 million deaths worldwide. ${ }^{3}$ COVID-19 is associated with a variable prognosis. Considering rapidly escalating number of cases, early 
risk stratification, prediction and evaluation of critical illness of COVID-19 is crucial and essential for improvement of the prognosis. The applicability of some widely used severity score for pneumonia, such as CURB-65, has been shown to be slightly scarce. ${ }^{4}$ Hence, the need for novel reliable and convenient prognostic biomarkers or predictors of COVID-19 has been highlighted, especially under the circumstances of limited medical resources.

Recently, the simple and accurate blood urea nitrogen (BUN) to serum albumin ratio (BAR), which results from the quotient between BUN and albumin, was introduced. Previous studies have demonstrated its utility as an independent prognostic indicator or significant predictor for mortality or intensive care in various diseases, including community-acquired pneumonia, Escherichia coli bacteremia, gastrointestinal bleeding, etc. ${ }^{5-7}$ Related studies have also demonstrated that deceased or critical ill COVID-19 patients often had higher BUN but lower albumin compared with mild patients. ${ }^{8,9}$ Moreover, it has also been reported that BAR is a more reliable predictor than BUN and albumin levels for predicting mortality in COVID-19 patients in the emergency department recently. ${ }^{10}$ However, above studies still suffer from some limitations, such as single-center design, medium sample size, potential unadjusted confounding factors, etc.

There is still a knowledge gap in the available literature concerning the associations between BAR and distinct clinical outcomes, such as development of critical illness, in COVID-19 patients. More validations of previous conclusions about BAR, including optimal cut-off values and adjusted odds ratio (OR), in different regions are also warranted before it is widely used in clinical practice. In the present study, we sought to explore the prognostic value of the BAR, and further develop a prediction model based on BAR for critical illness in COVID-19 patients.

\section{Patients and Methods Study Design and Participants}

This was a retrospective, multicenter, observational study on adult hospitalized COVID-19 patients from three COVID-19 designated hospitals in Wuhan city, Hubei province (Wuhan Red Cross hospital, People's Hospital of Wuhan University, Tongji Hospital of Huazhong University of Science and Technology), thirty-six COVID-19 designated hospitals in Sichuan province and fifteen COVID-19 designated hospitals in Guizhou province, China between January 14 and March 9, 2020. It was performed according to the amended Declaration of Helsinki and approved by the West China Hospital of Sichuan University Biomedical Research Ethics Committee (No.2020-272). Written informed consent was waived owing to retrospective observational nature. All patient data was maintained with confidentiality.

All patients enrolled in this study were laboratoryconfirmed COVID-19 according to WHO interim guidance, which was defined as positive result of the nucleic acid of SARS-CoV-2 by real-time reverse-transcription polymerase chain reaction (RT-PCR) of nasopharyngeal swab or oropharyngeal swab samples. ${ }^{11}$ The exclusion criteria were as follows: (1) under 18 years; (2) being pregnant; (3) died or discharged or had incomplete baseline clinical data within 24 hours after admission; (4) advanced liver disease; (5) advanced kidney disease: end stage renal failure or hemodialysis. (6) had malignancy; (7) chronic hematological diseases; (8) chronic immunosuppression: human immunodeficiency virus infection, chemotherapy, or other immunosuppressive therapy.

\section{Study Outcomes and Data Collection}

Primary outcome of interest was critical illness, which was defined as the composite endpoint of admission to the intensive care unit (ICU), need for invasive mechanical ventilation (IMV), or death. They have all been proved to be serious outcomes of COVID-19 and this composite measure has been used in previous similar studies of COVID-19. ${ }^{12,13}$

Clinical data elements collected included demographic characteristics, basic vital signs, symptoms and signs, comorbidities, chest computed tomography (CT) scan images and laboratory examinations at admission or within 24 hours after admission to hospitals. Specifically, the BUN count was divided by albumin count to obtain the BAR. Two experienced doctors reviewed the medical records and completed the data collection independently. Any disagreement was resolved by third doctor and team discussion until consensus reached.

\section{Statistical Analysis}

Data were analyzed by using IBM SPSS Statistics version 23.0 and $\mathrm{R}$ software 4.0.2. The data were tested by the Kolmogorov-Smirnov normality test and Bartlett's test for homogeneity of variance. The non-normally distributed continuous variables were described as median with interquartile range (IQR) while the normally distributed continuous variables were expressed as mean \pm standard 
deviation values, and categorical variables were shown as frequencies and percentages. Differences in continuous variables between groups were compared using MannWhitney $U$-test, while the Fisher's exact test and chisquare analysis were used to examine differences in categorical values.

The patients were divided into two groups according to the presence or absence of critical illness. Variables with $\mathrm{P}<0.10$ were included in univariate and multivariate logistic regression analysis to identify independent risk factors of critical illness. Only variables considered to be related to poor clinical outcomes in previous reports or in clinical practice, and to be independent with other variables were included into multivariate logistic regression analysis to avoid overfitting. The results were reported as OR and $95 \%$ confidence interval $(95 \% \mathrm{CI})$. Receiver operating characteristic (ROC) curve analysis evaluated predictive capacities of BUN, albumin and BAR on critical illness. Meanwhile, as the most common used severity score for pneumonia, the CURB-65 comprising 5 variables, attributing 1 point for each item: new onset confusion, urea $>7 \mathrm{mmol} / \mathrm{L}$, respiratory rate $\geq 30 /$ minute, systolic blood pressure $<90 \mathrm{mmHg}$ and/or diastolic blood pressure $\leq 60 \mathrm{mmHg}$ and age $\geq 65$ years, has been also calculated. Then, ROC curve of CURB-65 score was also performed to compare their predictive powers. ${ }^{4}$ These results were reported as area under the curve (AUC) and 95\% CI. The cut-off value of BAR was established by maximizing the Youden index. ${ }^{14}$ Then, all patients were divided into higher BAR group and lower BAR group in terms of cutoff value to examine increased BAR as a predictor of critical illness. The subgroup analyses were conducted according to area, age, sex, history of alcohol use or smoking and comorbidity to verify the robustness of overall results. The OR and 95\% CI of critical illness in patients having higher BAR compared with those having lower BAR in each subgroup was reported. The prediction model was established through the "rms" package in $\mathrm{R}$ based on results of multivariate Logistic regression. The goodness of fit of prediction model was evaluated via concordance index (C index) with $95 \%$ CI. ${ }^{15}$ Then, a nomogram was visually established based on the prediction model with the highest $\mathrm{C}$ index. ${ }^{16}$ The nomogram was also assessed by calibration curve and decision curve analysis (DCA) to further test its performance and accuracy. ${ }^{17,18}$ Significance was defined at the $\mathrm{P}<0.05$ level.

\section{Results}

\section{Baseline Patient Characteristics}

A total of 1485 patients confirmed with COVID-19 were included in the study. Finally, 115 of them were excluded according to the exclusion criteria (Figure 1) and 1370 were included. Totally $113(8.2 \%)$ patients eventually developed critical illness. The numbers and ratios of cases with critical illness were 93/977 (9.5\%) in Wuhan city, 15/292 (5.1\%) in Sichuan province, and 5/101 (5\%) in Guizhou province, respectively. Among them, 99 (7.2\%) patients were admitted into ICU and $51(3.7 \%)$ patients needed for IMV. Finally, 44 (3.2\%) patients died. Compared with patients without critical illness, the critical illness group had higher median age (67 vs 54 years, $\mathrm{P}<0.001)$, respiratory rate $(25$ vs 17 breath $/ \mathrm{min}$, $\mathrm{P}<0.001)$, rate of unconscious patients $(8.8 \%$ vs $0.2 \%$, $\mathrm{P}<0.001)$, total bilirubin $(15.2 \mu \mathrm{mol} / \mathrm{L}$ vs $9.7 \mu \mathrm{mol} / \mathrm{L}$, $\mathrm{P}<0.001)$, but lower lymphocyte counts $\left(0.75 \times 10^{9} / \mathrm{L}\right.$ vs $\left.1.21 \times 10^{9} / \mathrm{L}, \mathrm{P}<0.001\right)$, etc. Detailed comparisons of baseline characteristics are shown in Table 1.

Meanwhile, patients with critical illness had significantly increased BUN (16.5 vs $12.1 \mathrm{mg} / \mathrm{dL}, \mathrm{P}<0.001)$ and BAR (4.72 $\mathrm{mg} / \mathrm{g}$ vs $3.05 \mathrm{mg} / \mathrm{g}, \mathrm{P}<0.001$ ), but decreased albumin $(3.65 \mathrm{~g} / \mathrm{dL}$ vs $3.91 \mathrm{~g} / \mathrm{dL}, \mathrm{P}<0.001)$ than that without critical illness (Figure 2A). Patients were further divided into four groups according to interquartile range of BAR: under $2.42 \mathrm{mg} / \mathrm{g}, 2.42-3.08 \mathrm{mg} / \mathrm{g}, 3.09-3.79 \mathrm{mg} / \mathrm{g}$, and above $3.80 \mathrm{mg} / \mathrm{g}$. The rates of critical illness significantly increased with elevated baseline BAR (Figure 2B).

\section{Prognostic Value of BAR}

The variables with $\mathrm{P}<0.10$ were included in univariate Logistic regression analysis and 24 variables were identified as potential risk factors. Then, only 14 variables were included into multivariate Logistic regression analysis after we have manually excluded factors, which were not independent with others or had little clinical significance. Finally, baseline age (OR: 1.031; 95\% CI: 1.014, 1.049; $\mathrm{P}<0.001$ ), respiratory rate (OR: $1.063 ; 95 \% \mathrm{CI}: 1.009$, 1.120; $\mathrm{P}=0.022$ ), unconsciousness (OR: $40.078 ; 95 \% \mathrm{CI}$ : 5.992, 268.061; $\mathrm{P}<0.001)$, lymphocyte counts (OR: 0.352; 95\% CI: 0.204, 0.607; $\mathrm{P}<0.001$ ), total bilirubin (OR: 1.030; 95\% CI: $1.001,1.060 ; \mathrm{P}<0.001)$ and $\mathrm{BAR}(\mathrm{OR}$ : 1.319 ; $95 \%$ CI: $1.183,1.471 ; \mathrm{P}<0.001)$ were found to be independent risk factors for critical illness in COVID-19 patients. These detailed results are shown in Supplementary Table 1. 


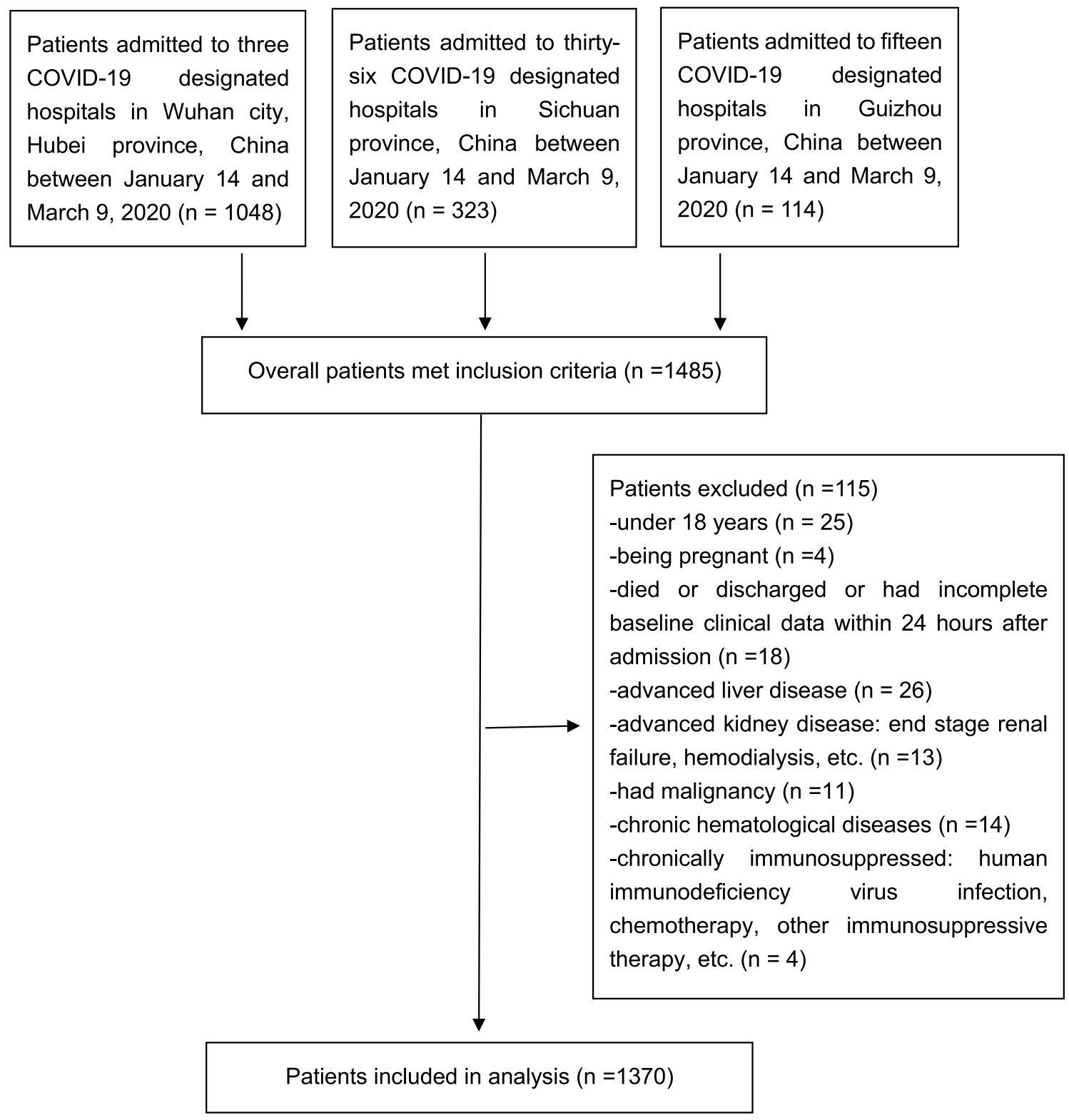

Figure I Study population.

A ROC curve was created to determine if baseline BAR was predictive of critical illness (Figure 3A). The AUC was 0.821 (95\% CI: 0.784, 0.858; $\mathrm{P}<0.01)$ for BAR, 0.783 (95\% CI: $0.743,0.823 ; \mathrm{P}<0.01)$ for $\mathrm{BUN}, 0.712$ (95\% CI: $0.664,0.760 ; \mathrm{P}<0.01)$ for albumin, and 0.744 (95\% CI: $0.690,0.798 ; \mathrm{P}<0.01$ ) for CURB-65, respectively. Therefore, BAR might be a better predictor than BUN, albumin and CURB-65 in COVID-19. The optimal cut-off value of BAR was $3.7887 \mathrm{mg} / \mathrm{g}$ according to maximal Youden index (sensitivity: 0. 690, specificity: 0.786; positive predictive value: 0.225 , negative predictive value: 0.966; positive likelihood ratio: 3.226 , negative likelihood ratio: 0.394$)$.
Among all included patients, odds of critical illness were significantly higher in those above the cut-off value compared to those below (OR: 3.302; 95\% CI: 1.959 , 5.565; $\mathrm{P}<0.001)$. The results of subgroup analysis were similar to pooled results (Figure 3B).

\section{Prediction Model for Critical Illness}

As a result, these above six predictors were included into the prediction model, as described in Figure 4. Each factor was assigned with a single score presented on the top line of nomogram. The total score of each patient is the sum of each single score. On the bottom of the nomogram, the probabilities of critical illness during hospitalization in 
Table I Comparisons of Clinical Characteristics Among COVID-19 Patients

\begin{tabular}{|c|c|c|c|c|}
\hline Variables & Overall $(n=1370)$ & $\begin{array}{l}\text { With Critical } \\
\text { Illness }(n=1 \text { |3) }\end{array}$ & $\begin{array}{l}\text { Without Critical } \\
\text { Illness }(n=\mid 257)\end{array}$ & $P$ value \\
\hline \multicolumn{5}{|l|}{ Demographic Characteristics } \\
\hline Sex (male) & $628(45.8)$ & $70(61.9)$ & $558(44.4)$ & $<0.001$ \\
\hline Age, years & $55(40,66)$ & $67(56,80)$ & $54(39,65)$ & $<0.001$ \\
\hline History of alcohol use & $240(17.5)$ & $24(21.2)$ & $216(17.2)$ & 0.277 \\
\hline Smoking history & $263(19.2)$ & $27(23.9)$ & $236(18.8)$ & 0.186 \\
\hline \multicolumn{5}{|l|}{ Vital Signs on Admission } \\
\hline Temperature $\left({ }^{\circ} \mathrm{C}\right)$ & $36.6(36.4,37)$ & $36.7(36.4,37.2)$ & $36.6(36.4,37)$ & 0.407 \\
\hline Heart rate (beat/min) & $84(78,94)$ & $84(78,97)$ & $84(78,94)$ & 0.682 \\
\hline Respiratory rate (breath/min) & $18(16,21)$ & $25(19,27)$ & $17(16,19)$ & $<0.001$ \\
\hline Systolic blood pressure $(\mathrm{mmHg})$ & $128(119,138)$ & $132(120,144)$ & $127(118,137)$ & 0.005 \\
\hline Diastolic blood pressure $(\mathrm{mmHg})$ & $79(70,85)$ & $80(71,86)$ & $78(70,85)$ & 0.498 \\
\hline \multicolumn{5}{|l|}{ Symptoms and Signs } \\
\hline Fever & $884(64.5)$ & $84(74.3)$ & $800(63.6)$ & 0.023 \\
\hline Cough & $855(62.4)$ & $74(65.5)$ & $781(62.1)$ & 0.481 \\
\hline Hemoptysis & $35(2.6)$ & $3(2.7)$ & $32(2.5)$ & 1.00 \\
\hline Short of breath/dyspnea & $314(22.9)$ & $44(38.9)$ & $270(21.5)$ & $<0.001$ \\
\hline Weakness/fatigue & $462(33.7)$ & $46(40.7)$ & $416(33.1)$ & 0.101 \\
\hline Sore throat/pharyngalgia & $89(6.5)$ & $9(8)$ & $80(6.4)$ & 0.509 \\
\hline Rhinorrhea & $28(2)$ & $\mathrm{I}(0.9)$ & $27(2.1)$ & 0.574 \\
\hline Wheeze & $132(9.6)$ & $21(18.6)$ & III(8.8) & 0.001 \\
\hline Stuffy nose & $21(1.5)$ & $0(0)$ & $21(1.7)$ & 0.325 \\
\hline Chest pain/distress & $257(18.8)$ & $26(23)$ & $231(18.4)$ & 0.227 \\
\hline Muscle ache/myalgia & $100(7.3)$ & $9(8)$ & $91(7.2)$ & 0.777 \\
\hline Arthralgia & $19(1.4)$ & $5(4.4)$ & $14(1.1)$ & 0.014 \\
\hline Headache & $65(4.7)$ & $6(5.3)$ & $59(4.7)$ & 0.768 \\
\hline Unconsciousness & $12(0.9)$ & $10(8.8)$ & $2(0.2)$ & $<0.001$ \\
\hline Stomachache & $20(1.5)$ & $5(4.4)$ & $15(1.2)$ & 0.02 \\
\hline Nausea/vomiting & $39(2.8)$ & $4(3.5)$ & $35(2.8)$ & 0.867 \\
\hline Diarrhea & $134(9.8)$ & $13(11.5)$ & $|2|(9.6)$ & 0.52 \\
\hline \multicolumn{5}{|l|}{ Comorbidities } \\
\hline Chronic heart disease & $115(8.4)$ & $20(17.7)$ & $95(7.6)$ & $<0.001$ \\
\hline Asthma & $9(0.7)$ & $0(0)$ & $9(0.7)$ & 1.00 \\
\hline COPD & $42(3.1)$ & $7(6.2)$ & $35(2.8)$ & 0.084 \\
\hline Chronic neural disease & $18(1.3)$ & $4(3.5)$ & $14(1.1)$ & 0.082 \\
\hline Diabetes mellitus & $191(13.9)$ & $14(12.4)$ & $177(14.1)$ & 0.619 \\
\hline Dementia & $18(1.3)$ & $3(2.7)$ & $15(1.2)$ & 0.381 \\
\hline Stroke history & $22(1.6)$ & $4(3.5)$ & $18(1.4)$ & 0.188 \\
\hline Hypertension & $386(28.2)$ & $46(40.7)$ & $340(27)$ & 0.002 \\
\hline \multicolumn{5}{|l|}{ Laboratory Examinations } \\
\hline White blood cell, $\times 10^{9} / \mathrm{L}$ & $5.5(4.36,6.94)$ & $6.5 \mathrm{I}(5.42,9.44)$ & $5.5(4.33,6.79)$ & $<0.001$ \\
\hline Hemoglobin, g/L & $127(116,140)$ & $124(107,143)$ & $128(118,140)$ & 0.17 \\
\hline Platelet counts, $\times 10^{9} / \mathrm{L}$ & $205(163,263)$ & $200(146,223)$ & $206(164,265)$ & 0.001 \\
\hline Lymphocyte counts, $\times 10^{9} / \mathrm{L}$ & $1.19(0.90,1.67)$ & $0.75(0.48,1.19)$ & $1.21(0.94,1.70)$ & $<0.001$ \\
\hline Neutrophil counts, $\times 10^{9} / \mathrm{L}$ & $3.47(2.59,4.73)$ & $4.93(3.47,8.27)$ & $3.47(2.55,4.50)$ & $<0.001$ \\
\hline Eosinophils, $\times 10^{9} / \mathrm{L}$ & $0.03(0.01,0.09)$ & $0.01(0.01,0.04)$ & $0.03(0.01,0.09)$ & $<0.001$ \\
\hline Basophils, $\times 10^{9} / \mathrm{L}$ & $0.01(0.01,0.03)$ & $0.01(0.01,0.02)$ & $0.01(0.01,0.03)$ & 0.141 \\
\hline Monocyte count, $\times 10^{9} / \mathrm{L}$ & $0.43(0.32,0.55)$ & $0.4 \mathrm{I}(0.27,0.50)$ & $0.43(0.33,0.56)$ & 0.004 \\
\hline D-dimer, mg/L & $0.57(0.35,0.93)$ & I. $18(0.57,3.39)$ & $0.57(0.32,0.82)$ & $<0.001$ \\
\hline
\end{tabular}

(Continued) 
Table I (Continued).

\begin{tabular}{|c|c|c|c|c|}
\hline Variables & Overall $(n=1370)$ & $\begin{array}{l}\text { With Critical } \\
\text { IIIness }(n=\mid 13)\end{array}$ & $\begin{array}{l}\text { Without Critical } \\
\text { Illness }(n=\mid 257)\end{array}$ & $P$ value \\
\hline Fibrinogen, $g / L$ & $3.56(2.94,4.32)$ & $3.69(3.41,5.4)$ & $3.56(2.9 I, 4.21)$ & $<0.001$ \\
\hline APTT, s & $27.9(26.8,32.1)$ & $28(27.5,31.9)$ & $27.8(26.7,32.1)$ & 0.495 \\
\hline PT, s & $12.1(11.7,12.9)$ & $12.7(12,13.7)$ & $12(\mid 1.6,12.8)$ & $<0.001$ \\
\hline INR & $1.03(0.97,1.06)$ & $1.06(1.03,1.15)$ & $1.03(0.96,1.05)$ & $<0.001$ \\
\hline Total bilirubin, $\mu \mathrm{mol} / \mathrm{L}$ & $9.9(8.2,14.3)$ & $15.2(9.0,19.2)$ & $9.7(8.2,13.7)$ & $<0.001$ \\
\hline ALT, IU/L & $24(17,36)$ & $24(18,40)$ & $24(17,35)$ & 0.541 \\
\hline AST, IU/L & $24.2(20,32)$ & $25(22,38.6)$ & $24(20,32)$ & 0.004 \\
\hline Total protein, $g / L$ & $64.6(61.1,69.9)$ & $63.1(57.8,65.3)$ & $64.6(61.5,70.1)$ & $<0.001$ \\
\hline Albumin, g/dL & $3.91(3.63,4.26)$ & $3.65(3.17,3.91)$ & $3.91(3.67,4.29)$ & $<0.001$ \\
\hline Globulin, g/L & $25.2(23.2,29.4)$ & $25.6(24.1,29.6)$ & $25.2(23.1,29.4)$ & 0.511 \\
\hline Triglyceride, $\mathrm{mmol} / \mathrm{L}$ & $1.23(1.09,1.48)$ & $1.24(1.13,1.48)$ & $1.23(1.08,1.47)$ & 0.769 \\
\hline Glucose, $\mathrm{mmol} / \mathrm{L}$ & $5.60(5.01,6.46)$ & $5.67(5.52,7.67)$ & $5.59(4.98,6.38)$ & $<0.001$ \\
\hline BUN, mg/dL & $\mid 2.1(9.78, \mid 4.57)$ & $16.5(12.1,25.4)$ & $12.1(9.52, \mid 4.02)$ & $<0.001$ \\
\hline Creatinine, $\mu \mathrm{mol} / \mathrm{L}$ & $62(53,72)$ & $63(55,73)$ & $62(53,72)$ & 0.795 \\
\hline Uric acid, umol/L & $263(218,319)$ & $267(238,326)$ & $260(215,319)$ & 0.149 \\
\hline C-reactive protein, $\mathrm{mg} / \mathrm{L}$ & $10(4,16)$ & $10.4(5.6,19)$ & $9.5(4,18.8)$ & 0.072 \\
\hline Procalcitonin, $\mu \mathrm{g} / \mathrm{L}$ & $0.05(0.04,0.06)$ & $0.06(0.05,0.06)$ & $0.05(0.04,0.06)$ & 0.35 \\
\hline $\mathrm{BAR}, \mathrm{mg} / \mathrm{g}$ & $3.09(2.42,3.80)$ & $4.72(3.35,7.55)$ & $3.05(2.35,3.63)$ & $<0.001$ \\
\hline \multicolumn{5}{|l|}{ Chest CT scan images } \\
\hline Consolidation & $190(13.9)$ & $17(15)$ & $173(\mid 3.8)$ & 0.706 \\
\hline Ground-glass opacity & $1116(81.5)$ & $87(77)$ & $1029(81.9)$ & 0.202 \\
\hline Paving & $7(0.5)$ & $0(0)$ & $7(0.6)$ & 1.00 \\
\hline Fibrotic & $293(21.4)$ & $17(15)$ & $276(22)$ & 0.086 \\
\hline Effusion & $50(3.6)$ & $4(3.5)$ & $46(3.7)$ & 1.00 \\
\hline
\end{tabular}

Note: Data are shown as median with interquartile range (IQR) for continuous variables or number with percentage for categorical variables.

Abbreviations: COVID-19, coronavirus disease 2019; critical illness, admitted into intensive care unit, invasive mechanical ventilation or death; n, numbers; COPD, chronic obstructive pulmonary disease; APTT, activated partial thromboplastin time; PT, prothrombin time; INR, international normalized ratio; ALT, alanine aminotransferase; AST, aspartate aminotransferase; BUN, blood urea nitrogen; BAR, blood urea nitrogen to serum albumin ratio; CT, computed tomography.

patients with COVID-19 were predicted in terms of the total scores.

By using bootstrap method, the $\mathrm{C}$ index was 0.9031125 (95\% CI: 0.8720542, 0.9341708). In Figure 5A, the calibration curve did not deviate from the reference line significantly. There was a good consistency between the predicted values by nomogram and actual observed values. The bias-corrected C index was 0.8984114. The DCA was performed to evaluate clinical applicability of prediction model in Figure 5B. The DCA demonstrated that the nomogram had good overall net benefits within a wide range of threshold probabilities. The nomogram could improve patient outcomes in clinical practice.

\section{Discussion}

In the present study, we found that BAR at hospital admission is an independent predictor for risk of critical illness in COVID-19 patients on subsequent hospital days, with superior performance than CURB-65. It is simple to calculate and could be employed as early as patient presentation in routine clinical practice. Furthermore, the nomogram including BAR provides clinicians with an easily obtainable tool to facilitate accurate evaluation of prognosis in COVID-19 patients. Our findings are of relevance for clinical decision-making in the COVID-19 pandemic.

Similar prognostic performances of BAR in various diseases have been observed frequently in recent years. At first, for infectious diseases, Ugajin et al found elevated BAR was an independent predictor of mortality (OR: 1.10, 95\% CI: 1.01-1.20) and need for intensive care (OR: 1.27, 95\% CI: $1.09-1.47$ ) in community-acquired pneumonia; ${ }^{5}$ Zou et al revealed BAR was an independent predictor of 30-day mortality and intensive case requirement in Escherichia coli bacteremia. ${ }^{6}$ Other researchers further demonstrated that increased BAR was a potentially useful 

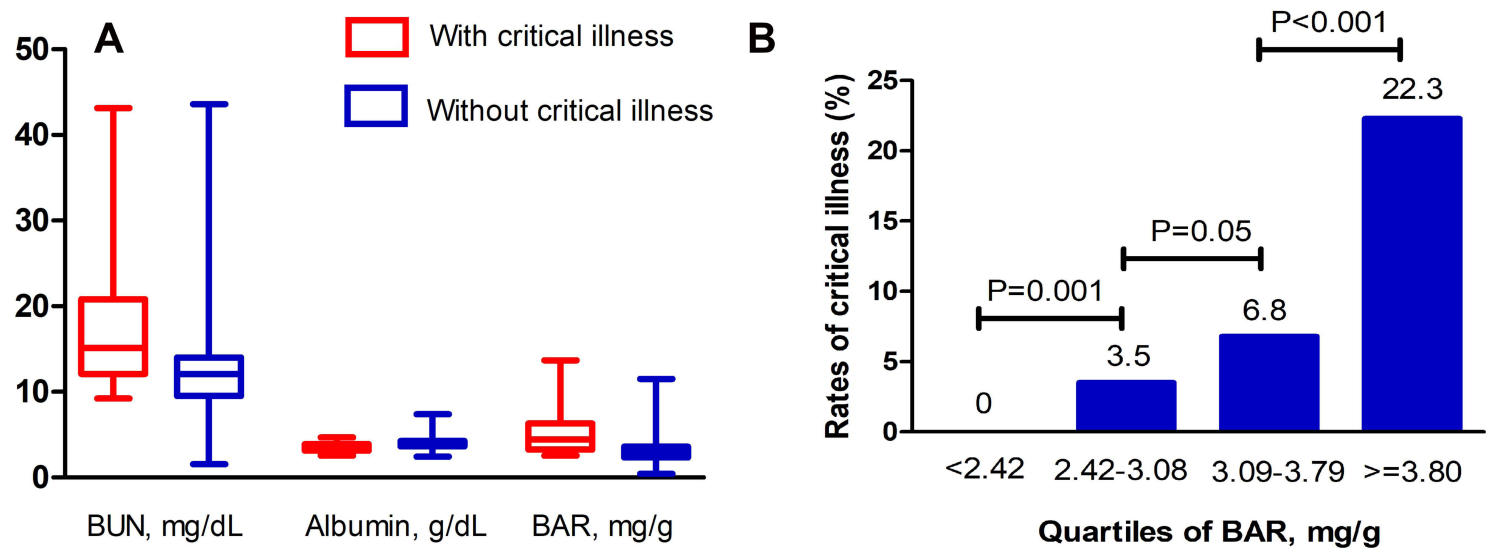

Figure 2 (A) The BUN, albumin and BAR values within 24 hours after admission in COVID-19 patients with or without critical illness. (B) Comparisons of the rates of critical illness among quartiles of BAR in COVID-19 patients. P values were calculated by Chi Square analysis.

Abbreviations: BUN, blood urea nitrogen; BAR, blood urea nitrogen to serum albumin ratio; COVID-19, coronavirus disease 2019.

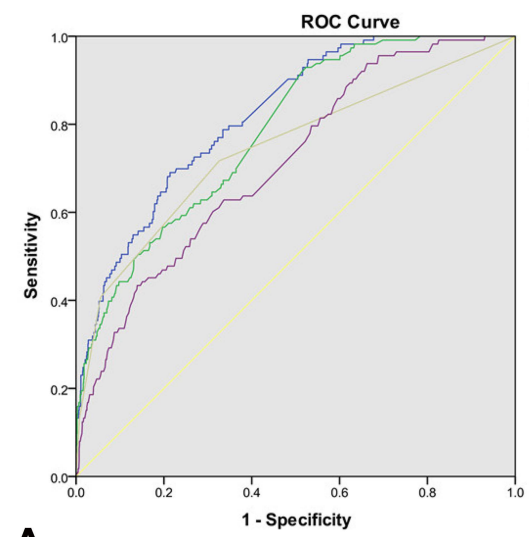

A

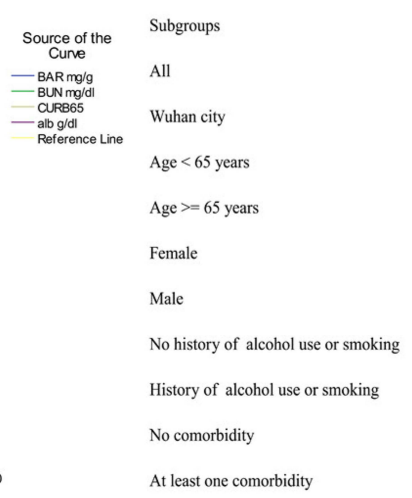

$\mathbf{B}$

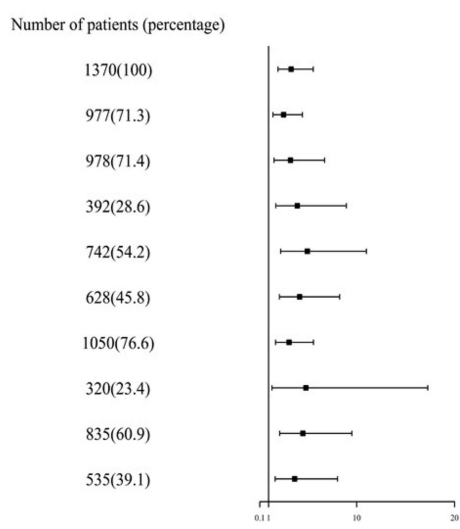

Figure 3 (A) ROC curve of BAR, BUN, albumin and CURB-65 for prediction of critical illness in COVID-19 patients. The AUC were 0.82I (95\% CI: 0.784, 0.858; P<0.0I) for BAR, 0.783 ( $95 \% \mathrm{Cl}$ : 0.743, 0.823; P<0.0I) for BUN, 0.712 (95\% Cl: 0.664, 0.760; P<0.0I) for albumin, and 0.744((95\% Cl: 0.690, 0.798; P<0.0I) for CURB-65, respectively. The optimal predictive cut-off value of BAR was $3.7887 \mathrm{mg} / \mathrm{g}$ (specificity: 0. 690; sensitivity: 0.786). (B) Subgroup analysis for OR of COVID-I9 patients with BAR $\geq 3.7887 \mathrm{mg} / \mathrm{g}$ versus BAR $<3.7887 \mathrm{mg} / \mathrm{g}$ stratified by area, age, sex, history of drinking or smoking, and comorbidity. P values were calculated by multivariate Logistics regression analysis.

Abbreviations: ROC, receiver operating characteristic; BAR, blood urea nitrogen to serum albumin ratio; COVID-19, coronavirus disease 20I9; AUC, area under the curve; OR, odds ratios; $95 \% \mathrm{Cl}, 95 \%$ confidence interval.

prognostic factor of mortality within 28 days in aspiration pneumonia patients and 30-day mortality in hospitalacquired pneumonia (hazard ratio (HR): $3.871 ; 95 \% \mathrm{CI}$ : 2.174-6.893). ${ }^{19,20}$ Then, Bae et al also found BAR was a significant predictor of ICU admission and in-hospital mortality in acute gastrointestinal bleeding patients. ${ }^{7}$ Lastly, Dundar et al concluded that BAR $>6.25$ was an independent predictor of in-hospital mortality in all older emergency department patients (aged 65 and over). ${ }^{21}$ The AUC of BAR in above studies varied from 0.69 to 0.83 . As an easy and convenient parameter, it is not affected by individual subjectivity from different clinicians and might be also a promising predictor in COVID-19. As
Küçükceran reported, the AUC of BAR in COVID-19 was 0.809 and $\mathrm{BAR}>3.9 \mathrm{mg} / \mathrm{g}$ was associated with increased in-hospital mortality (OR: 10.448; 95\% CI: $5.562-19.626) .{ }^{10}$ It is noted that the OR was calculated by using univariate logistic regression analysis. Furthermore, there is scarce data on the predictive value for critical illness of BAR in COVID-19. Finally, in the current study, the AUC is 0.82 and the multivariate OR of BAR > 3.7887 for critical illness is 3.302 (95\% CI: 1.959, 5.565), which means our results maintain the previously demonstrated prognostic utility of BAR in COVID-19 patients. However, it should be noticed that BAR $\geq$ $3.7887 \mathrm{mg} / \mathrm{g}$ was not identified as an independent 


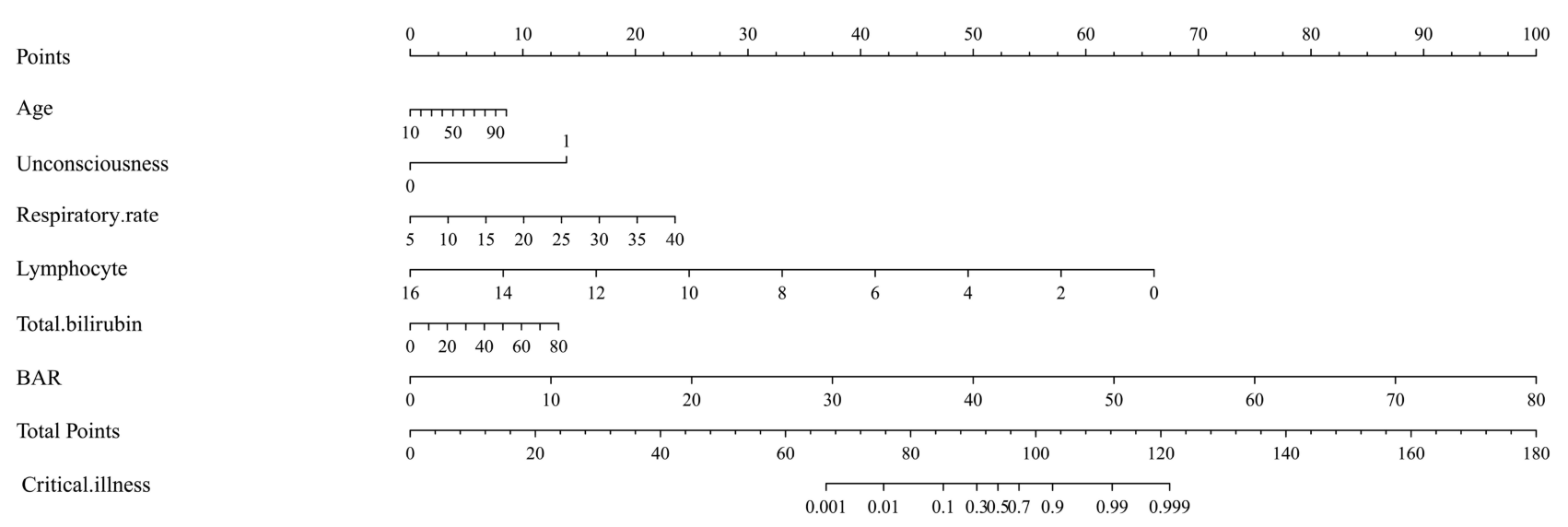

Figure 4 Predictive nomogram for critical illness in COVID-19 patients. Age (years); Unconsciousness (I: yes, 0: no); Respiratory rate (breath/min); Lymphocyte $\left(\times 10^{9} / \mathrm{L}\right)$; Total bilirubin $(\mu \mathrm{mol} / \mathrm{L})$; BAR $(\mathrm{mg} / \mathrm{g})$.

Abbreviations: COVID-19, coronavirus disease 2019; BAR, blood urea nitrogen to serum albumin ratio.

\section{A}

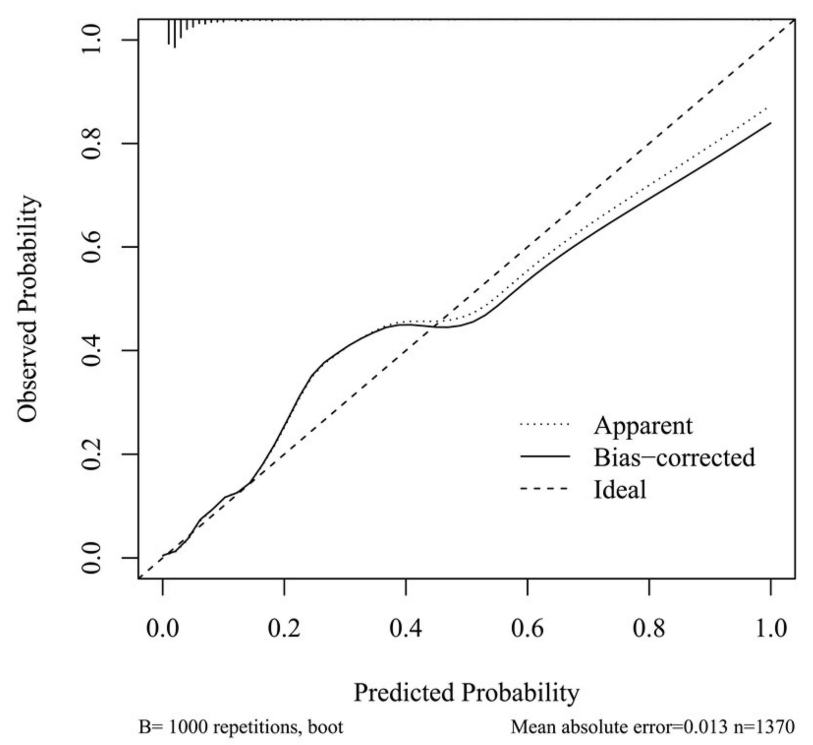

Figure 5 (A) The calibration curve of nomogram. (B) The DCA of nomogram. Abbreviation: DCA, decision curve analysis.

predictor of critical illness in Sichuan province or Guizhou province. It might be due to insufficient sample size because the numbers of cases with critical illness in these two areas were far less than that in Wuhan city at the beginning of 2020 . Therefore, the predictive performance of BAR still needs more validation in different regions.

The reasons and pathophysiological mechanisms responsible for prognostic values of BAR are still unclear. Urea is synthesized in liver via protein catabolism and blood urea is freely filtered at the glomerulus and undergoes tubular reabsorption. BUN could reflect

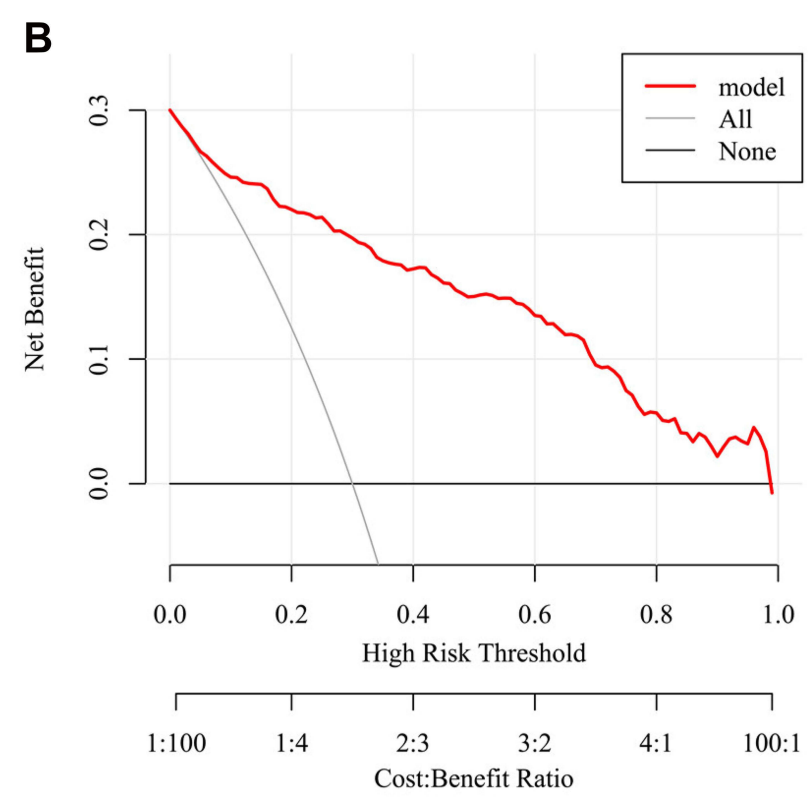

the complex interrelation among nutritional status, protein metabolism and renal condition of the patient. Previous renal histopathological analysis in 26 autopsies of patients with COVID-19 revealed that factors contributing to acute kidney injury included direct virulence of SARS-CoV-2 through high expression and upregulation of angiotensin-converting enzyme type II (ACEII) receptor in the kidneys, systemic hypoxia, abnormal coagulation, and possible drug or hyperventilation-relevant rhabdomyolysis. $^{22}$ The virus can activate the reninangiotensin-aldosterone system (RAAS) and cause renal vasoconstriction, slowing of tubular flow and decrease in 
glomerular ultrafiltration, leading to reduced BUN excretion and increased absorption of water and sodium in the kidney tubules, and passive reabsorption of BUN. ${ }^{23}$ It should be noted that dehydration is common in patients with pneumonia. In the dehydrated condition, reabsorption of urea by the kidneys is also increased. ${ }^{5,19,20}$ Recently two meta-analyses of COVID-19 demonstrated that $13.7 \%$ (95\% CI 5.5-21.9\%) of patients had elevated serum BUN levels and higher level of BUN was associated with a significant increase in fatality (mean difference (MD): $4.07 \mathrm{mmol} / \mathrm{L}, 95 \% \mathrm{CI}: 3.33-4.81$ ) and severe infection (MD: $2.12 \mathrm{mmol} / \mathrm{L}, 95 \%$ CI: 1.74-2.50). ${ }^{24,25}$

As the most abundant circulating protein and a wellknown plasma-expander, serum albumin also plays a key role in the neutralization of free radicals and exogenous or endogenous substances, antioxidation, immune-modulation, anti-inflammatory processes and endothelial stabilization. ${ }^{26}$ Serum albumin concentration can be reduced by malnourishment, inflammation, hepatocellular injury, renal losses, etc. Previous studies have demonstrated that the inflammatory reaction was the main reason for depressed serum albumin levels and there was no correlation between the serum albumin levels and the nutritional measurements in elderly patients with pneumonia. ${ }^{27}$ In COVID-19, the predictive values of hypoalbuminemia might be explained by several characteristics of critical illness, such as increased capillary leakage, tissue ischemia, reperfusion injury, intense systemic inflammatory response, diminished immunological response, etc. ${ }^{28}$ A meta-analysis of 11 studies with 910 patients indicated that the weighted mean serum albumin on admission was statistically significant (MD: $-0.56 \mathrm{~g} / \mathrm{dL} ; 95 \% \mathrm{CI}:-0.69$ to $-0.42 \mathrm{~g} / \mathrm{dL}$ ) in severe and non-severe COVID-19 group, and the hypoalbuminemia status was related to increased risk of severe COVID-19 (OR: 12.6; 95\% CI: 7.5-21.1). ${ }^{29}$

Previous reports have also proposed some prediction models for mortality or severity of COVID-19, in which the increased BUN and decreased albumin levels are in agreement with our results. Altschul et al developed a COVID-19 severity score consisting of BUN, age, oxygen saturation, etc. (AUC in training cohort: 0.824; validation cohort: 0.798). ${ }^{30}$ Another nomogram including BUN, albumin, age, bilirubin, etc. could be used for early identifying severe COVID-19 (AUC in training cohort: 0.912; validation cohort: 0.853). ${ }^{31}$ Besides, a machine learning model using BUN, albumin and several other serum chemistry laboratory parameters was developed for the prediction of death of COVID-19 (91\% sensitivity, 91\% specificity, AUC: 0.93). ${ }^{32}$ The BAR and nomogram in our study have comparative or superior predictive performances compared with above models. In this regard, our findings have novelty and clinical utility.

Our results showed that the rate of critical illness was $8.2 \%$, which was similar or slightly lower than to that reported by previous studies..$^{12,33,34}$ One possible reason is that we have excluded the COVID-19 patients with advanced comorbidities, such as liver disease, kidney disease, malignancy, hematological diseases and immunosuppressed to ensure the accuracy of BUN and albumin levels. However, as previously reported these patients are often more likely to develop critical illness. In addition, we found that age, unconsciousness, respiratory rate, lymphocyte and total bilirubin made the highest contribution to the prediction of critical illness after adjustment for demographic and clinical parameters. This result is consistent with findings form previous reports..$^{7,8,12,13}$ These observations confirmed the representativeness of our sampling population and the accuracy of our results. Therefore, the present results support the measurement of baseline BAR in hospitalized patients with COVID-19. Future clinical research efforts could examine its prognostic values among different medical settings or other specific COVID19 populations, such as children, pregnant women, immunocompromised patients with comorbidities, etc. Additional studies including large numbers of patients are needed to calibrate the optimal cutoff value of BAR and to validate the nomogram. More researches demonstrating the clinical course and pathophysiology of COVID-19 is of great importance for routine clinical use of BAR.

Our current study has several limitations. Firstly, this study was a retrospective analysis and the cohort included a limited number of patients. We only included patients from three provinces in China without further external validation. The nomogram was derived from particular periods and places where data were collected. Secondly, the results might have been affected by some unadjusted confounders, including the heterogeneities of drugs before admission and treatments among different medical centers. Thirdly, we failed to record changes of BAR after admission or make follow-up after discharge due to scarcity of relative information.

\section{Conclusion}

In conclusion, elevated BAR at admission is an independent risk factor for critical illness and appears to be a promising predictor in COVID-19 patients. The predictive nomogram including age, unconsciousness, 
respiratory rate, lymphocyte, total bilirubin and BAR for critical illness has superior predictive capacity. Our results need more validations.

\section{Abbreviations}

BAR, blood urea nitrogen to serum albumin ratio; COVID-19, coronavirus disease 2019; BUN, blood urea nitrogen; ICU, intensive care unit; IMV, invasive mechanical ventilation; ROC, receiver operating characteristic; OR, odds ratio; $95 \%$ CI, 95\% confidence interval; SARSCoV-2, severe acute respiratory syndrome coronavirus 2 ; WHO, World Health Organization; RT-PCR, reversetranscription polymerase chain reaction; $\mathrm{CT}$, computed tomography; AUC, area under the curve; DCA, decision curve analysis; ACEII, angiotensin-converting enzyme type II; RAAS, renin-angiotensin-aldosterone system; $\mathrm{MD}$, mean difference.

\section{Data Sharing Statement}

The datasets used and/or analyzed during the current study are available from the corresponding author on reasonable request.

\section{Ethics Approval and Informed Consent}

This study was approved by the West China Hospital of Sichuan University Biomedical Research Ethics Committee (No.2020-272). Written informed consent was waived due to retrospective observational design. All patient data was maintained with confidentiality.

\section{Author Contributions}

All authors contributed to data analysis, drafting or revising the article, have agreed on the journal to which the article will be submitted, gave final approval of the version to be published, and agreed to be accountable for all aspects of the work.

\section{Funding}

This work was supported by the Emergency Response Project for New Coronavirus of Science and Technology Department of Sichuan Provincial (2020YFS0005 and 2020YFS0009).

\section{Disclosure}

The authors report no conflicts of interest in this work.

\section{References}

1. Zhu N, Zhang D, Wang W, et al. A novel coronavirus from patients with pneumonia in China, 2019. $N$ Engl J Med. 2020;382 (8):727-733. doi:10.1056/NEJMoa2001017

2. WHO Director-General's opening remarks at the media briefing on COVID-19 - 11 March 2020. World Health Organization. Available from: https://www.who.int/director-general/speeches/detail/who-direc tor-general-s-opening-remarks-at-the-media-briefing-on-covid-1911-march-2020 . Accessed April 1, 2021.

3. COVID-19 Map. Johns Hopkins coronavirus resource center. Available from: https://coronavirus.jhu.edu/map.html. Accessed March 23, 2021.

4. Nguyen Y, Corre F, Honsel V, et al. Applicability of the CURB-65 pneumonia severity score for outpatient treatment of COVID-19. J Infect. 2020;81(3):e96-e98. doi:10.1016/j.jinf.2020.05.049

5. Ugajin M, Yamaki K, Iwamura N, Yagi T, Asano T. Blood urea nitrogen to serum albumin ratio independently predicts mortality and severity of community-acquired pneumonia. Int J Gen Med. 2012;5:583-589. doi:10.2147/IJGM.S33628

6. Zou XL, Feng DY, Wu WB, Yang HL, Zhang TT. Blood urea nitrogen to serum albumin ratio independently predicts 30-day mortality and severity in patients with Escherichia coli bacteraemia [published online ahead of print, 2020 Oct 12]. Med Clin (Barc). 2020;S0025-7753(20)30638-2. doi:10.1016/j.medcli.2020.06.060

7. Bae SJ, Kim K, Yun SJ, Lee SH. Predictive performance of blood urea nitrogen to serum albumin ratio in elderly patients with gastrointestinal bleeding. Am J Emerg Med. 2021;41:152-157. doi:10.1016/ j.ajem.2020.12.022

8. Ghahramani S, Tabrizi R, Lankarani KB, et al. Laboratory features of severe vs. non-severe COVID-19 patients in Asian populations: a systematic review and meta-analysis. Eur J Med Res. 2020;25 (1):30. doi:10.1186/s40001-020-00432-3

9. Izcovich A, Ragusa MA, Tortosa F, et al. Prognostic factors for severity and mortality in patients infected with COVID-19: a systematic review. PLoS One. 2020;15(11):e0241955. doi:10.1371/journal.pone. 0241955

10. Küçükceran K, Ayrancı MK, Girişgin AS, Koçak S, Dündar ZD. The role of the BUN/albumin ratio in predicting mortality in COVID-19 patients in the emergency department. Am $J$ Emerg Med. 2021;48:33-37. doi:10.1016/j.ajem.2021.03.090

11. World Health Organization. Clinical management of severe acute respiratory infection when COVID-19 is suspected: interim guidance; 2020. Available from: https://www.who.int/publications-detail/clinicalmanagement-of-severe-acute-respiratory-infection-when-novelcoronavirus-(ncov)-infection-is-suspected. Accessed August 11, 2021.

12. Liang W, Liang H, Ou L, et al. Development and validation of a clinical risk score to predict the occurrence of critical illness in hospitalized patients with COVID-19. JAMA Intern Med. 2020;180 (8):1081-1089. doi:10.1001/jamainternmed.2020.2033

13. Zheng Y, Xiao A, Yu X, et al. Development and validation of a prognostic nomogram based on clinical and CT features for adverse outcome prediction in patients with COVID-19. Korean J Radiol. 2020;21(8):1007-1017. doi:10.3348/kjr.2020.0485

14. Youden WJ. Index for rating diagnostic tests. Cancer. 1950;3 (1):32-35. doi:10.1002/1097-0142(1950)3:1<32::AIDCNCR2820030106 $>3.0 . C O ; 2-3$

15. Caetano SJ, Sonpavde G, Pond GR. C-statistic: a brief explanation of its construction, interpretation and limitations. Eur $J$ Cancer. 2018;90:130-132. doi:10.1016/j.ejca.2017.10.027

16. Iasonos A, Schrag D, Raj GV, Panageas KS. How to build and interpret a nomogram for cancer prognosis. J Clin Oncol. 2008;26 (8):1364-1370. doi:10.1200/JCO.2007.12.9791

17. Alba AC, Agoritsas T, Walsh M, et al. Discrimination and calibration of clinical prediction models: users' guides to the medical literature. JAMA. 2017;318(14):1377-1384. doi:10.1001/jama.2017.12126 
18. Vickers AJ, Elkin EB. Decision curve analysis: a novel method for evaluating prediction models. Med Decis Making. 2006;26 (6):565-574. doi:10.1177/0272989X06295361

19. Ryu S, Oh SK, Cho SU, et al. Utility of the blood urea nitrogen to serum albumin ratio as a prognostic factor of mortality in aspiration pneumonia patients. Am J Emerg Med. 2020;43:175-179.

20. Feng DY, Zhou YQ, Zou XL, et al. Elevated blood urea nitrogen-toserum albumin ratio as a factor that negatively affects the mortality of patients with hospital-acquired pneumonia. Can J Infect Dis Med Microbiol. 2019;2019:1547405. doi:10.1155/2019/1547405

21. Dundar ZD, Kucukceran K, Ayranci MK. Blood urea nitrogen to albumin ratio is a predictor of in-hospital mortality in older emergency department patients. Am J Emerg Med. 2021;46:349-354. doi:10.1016/j.ajem.2020.10.008

22. Su H, Yang M, Wan C, et al. Renal histopathological analysis of 26 postmortem findings of patients with COVID-19 in China. Kidney Int. 2020;98(1):219-227. doi:10.1016/j.kint.2020.04.003

23. Ok F, Erdogan O, Durmus E, Carkci S, Canik A. Predictive values of blood urea nitrogen/creatinine ratio and other routine blood parameters on disease severity and survival of COVID-19 patients. $J$ Med Virol. 2021;93(2):786-793. doi:10.1002/jmv.26300

24. Yang X, Jin Y, Li R, Zhang Z, Sun R, Chen D. Prevalence and impact of acute renal impairment on COVID-19: a systematic review and meta-analysis. Crit Care. 2020;24(1):356. doi:10.1186/s13054-02003065-4

25. Shao M, Li X, Liu F, Tian T, Luo J, Yang Y. Acute kidney injury is associated with severe infection and fatality in patients with COVID-19: a systematic review and meta-analysis of 40 studies and 24,527 patients. Pharmacol Res. 2020;161:105107. doi:10.1016/j.phrs.2020.105107

26. Caraceni P, Domenicali M, Tovoli A, et al. Clinical indications for the albumin use: still a controversial issue. Eur J Intern Med. 2013;24 (8):721-728. doi:10.1016/j.ejim.2013.05.015
27. Hedlund JU, Hansson LO, Ortqvist AB. Hypoalbuminemia in hospitalized patients with community-acquired pneumonia. Arch Intern Med. 1995;155(13):1438-1442. doi:10.1001/archinte.1995.00430130132014

28. Kheir M, Saleem F, Wang C, Mann A, Chua J. Higher albumin levels on admission predict better prognosis in patients with confirmed COVID-19. PLoS One. 2021;16(3):e0248358. doi:10.1371/journal. pone. 0248358

29. Aziz M, Fatima R, Lee-Smith W, Assaly R. The association of low serum albumin level with severe COVID-19: a systematic review and meta-analysis. Crit Care. 2020;24(1):255. doi:10.1186/s13054-02002995-3

30. Altschul DJ, Unda SR, Benton J, et al. A novel severity score to predict inpatient mortality in COVID-19 patients. Sci Rep. 2020;10 (1):16726. doi:10.1038/s41598-020-73962-9

31. Gong J, Ou J, Qiu X, et al. A tool for early prediction of severe coronavirus disease 2019 (COVID-19): a multicenter study using the risk nomogram in Wuhan and Guangdong, China. Clin Infect Dis. 2020;71(15):833-840. doi:10.1093/cid/ciaa443

32. Booth AL, Abels E, McCaffrey P. Development of a prognostic model for mortality in COVID-19 infection using machine learning. Mod Pathol. 2021;34(3):522-531. doi:10.1038/s41379-020-00700-X

33. Yang X, Yu Y, Xu J, et al. Clinical course and outcomes of critically ill patients with SARS-CoV-2 pneumonia in Wuhan, China: a single-centered, retrospective, observational study. Lancet Respir Med. 2020;8(5):475-481. doi:10.1016/S2213-2600(20)30079-5

34. Richardson S, Hirsch JS, Narasimhan M, et al. Presenting characteristics, comorbidities, and outcomes among 5700 patients hospitalized with COVID-19 in the new york city area. JAMA. 2020;323 (20):2052-2059. doi:10.1001/jama.2020.6775
International Journal of General Medicine

\section{Publish your work in this journal}

The International Journal of General Medicine is an international, peer-reviewed open-access journal that focuses on general and internal medicine, pathogenesis, epidemiology, diagnosis, monitoring and treatment protocols. The journal is characterized by the rapid reporting of reviews, original research and clinical studies across all disease areas. The manuscript management system is completely online and includes a very quick and fair peer-review system, which is all easy to use. Visit http://www.dovepress.com/ testimonials.php to read real quotes from published authors. 